\title{
O Status Atual do Ensino do Pensamento Computacional no Estado do Paraná
}

\author{
Carolina Andrade Presotto ${ }^{1}$, Daniel S. Kaster ${ }^{1}$ \\ ${ }^{1}$ Departamento de Computação - Universidade Estadual de Londrina (UEL) \\ Caixa Postal 10.011 - 86.057-970 - Londrina - PR - Brasil \\ carolyna-andradeahotmail.com, dskasterauel.br
}

\begin{abstract}
Teaching Computational Thinking (CT) has become essential for all citizens of the 21st century to enable people to solve problems and perform their daily activities using technological tools. The objective of this work is to investigate how the state of Paraná is being prepared to fulfill from 2021 what the National Common Curricular Base (BNCC) proposes about CT teaching and how schools from two important cities in the state have been teaching this subject. From a literature review, we observe that official documents that guide the state's education do not mention CT yet, even though the state has been offering CT in an extracurricular way in some public schools and providing free online training to teachers. It urges a systematization to broadly offer CT teaching in the state's schools, which is still in an early stage.
\end{abstract}

Resumo. O ensino do Pensamento Computacional (PC) tornou-se essencial para todos os cidadãos do século XXI para habilitar pessoas a resolver problemas e realizar suas atividades cotidianas usando ferramentas tecnológicas. O objetivo deste trabalho é investigar como o estado do Paraná está se preprando para atender, a partir de 2021, o que a Base Nacional Comum Curricular (BNCC) propõe sobre o ensino do PC e como escolas de duas cidades importantes do estado têm ensinado este conteúdo. A partir de uma revisão bibliográfica, observou-se que os documentos oficiais que regem a educação no estado ainda não mencionam o PC, embora o estado venha oferecendo PC de forma extracurricular em algumas escolas públicas e provendo treinamentos online gratuitos para professores. É necessária uma sistematização para um ensino abrangente do PC nas escolas do estado, que ainda encontra-se em um estágio inicial.

\section{Introdução}

O avanço tecnológico trouxe grandes mudanças em todos os setores da sociedade, exigindo da escola a revisão de seus currículos e métodos de ensino com vista a atender a realidade em que os alunos estão inseridos, preparando-os para se tornarem cidadãos críticos e prontos para atuar na sociedade. De acordo com Moran (2007), a educação busca e transmite referenciais do passado, olha para o hoje, ensinando o aluno a compreender a si mesmo e a sociedade em que vive e, ao mesmo tempo, o prepara para os desafios que virão futuramente.

Neste contexto, recentemente se intensificaram as discussões a respeito da necessidade do ensino do Pensamento Computacional (PC) na educação básica. 
Segundo Wing (2006), o PC é uma habilidade fundamental para todos, não somente para cientistas da computação. À leitura, escrita e aritmética, deveríamos incluí-lo como habilidade analítica fundamental. É crucial que a escola desenvolva o PC nos alunos, desde a educação básica, para que consigam atuar significativamente nesta sociedade cada vez mais tecnológica, que exige dos cidadãos mais do que apenas manusear artefatos eletrônicos. Dentro dos currículos da educação básica de alguns países, o PC "trata-se de pensar não somente na perspectiva do usuário que se apropria das tecnologias, mas daquele que planeja, desenha, desenvolve e apresenta à sociedade" (ARRUDA, 2017). Esta concepção torna o aluno um protagonista em sua sociedade, sendo capaz de criar tecnologias, ao invés de se limitar a ser um mero usuário.

O Pensamento Computacional na educação básica brasileira ainda é um assunto recente, iniciando suas discussões em meados de 2012. No ano de 2018, foi homologada a versão final da Base Nacional Comum Curricular (BNCC), que é o primeiro documento oficial dentre os documentos que regulamentam o ensino da educação básica nacional que reconhece o PC, propondo a implementação de soluções envolvendo diferentes tecnologias, como ferramentas para identificar, analisar, modelar e solucionar problemas complexos explorando o PC no cotidiano (BRASIL, 2018).

Particularmente, para o estado do Paraná, há poucas referências com respeito ao PC na educação básica do estado, em geral, apresentando resultados de pesquisas de campo em escolas públicas ou privadas que trabalham o tema de forma extracurricular, por meio de oficinas geralmente ministradas por graduandos de cursos de Computação, Informática ou Matemática. Neste contexto, a questão fundamental deste trabalho é: como o estado do Paraná está cumprindo o que está proposto na BNCC em relação ao ensino do PC?

O objetivo deste trabalho foi averiguar a presença do Pensamento Computacional na educação básica do estado do Paraná. Para isso, utilizou-se de pesquisa exploratória, tendo como procedimento técnico o levantamento bibliográfico. $\mathrm{O}$ trabalho sumariza como documentos oficiais que norteiam a educação básica tanto no Brasil quanto no estado do Paraná abordam o ensino do $\mathrm{PC}$ e discute a importância da formação inicial e continuada de professores para o ensino deste conteúdo. Por fim, apresenta uma análise da situação atual da presença do PC em escolas públicas do Paraná, de forma curricular ou extracurricular, de esforços governamentais e de projetos independentes para a disseminação do PC no estado.

Os principais resultados deste trabalho foram: (i) a constatação de que o termo Pensamento Computacional não aparece nos documentos oficiais que normatizam a educação do estado do Paraná; (ii) a verificação de que o PC também não aparece de forma explícita nas disciplinas ofertadas nas escolas de período integral e nem nos programas que a Secretaria de Estado da Educação e do Esporte do Paraná oferece de forma extracurricular; e (iii) que o Estado do Paraná está caminhando lentamente no que se refere a formação de professores para o ensino do PC nas escolas.

\section{Conceitos Fundamentais e Trabalhos Relacionados}

O termo Pensamento Computacional ganhou bastante destaque a partir da publicação do artigo Computational Thinking, de Wing (2006), no qual a autora apresentou o significado do termo e defendeu a importância de ser ensinado a todas as pessoas. De uma forma ampla, o conceito de Pensamento Computacional compreende 
processos de pensamento que envolvem a formulação de um problema, expressando suas soluções de tal forma que um computador ou humano possam executar de forma efetiva (WING, 2014).

A Sociedade Brasileira de Computação (SBC), em suas diretrizes para o ensino de Computação na educação básica, define o PC como sendo a "[...] capacidade de compreender, definir, modelar, comparar, solucionar, automatizar e analisar problemas (e soluções) de forma metódica e sistemática, através da construção de algoritmos" (SBC, 2019). Desta forma, o PC vem como uma habilidade a ser desenvolvida em todos os indivíduos, sendo útil tanto para a vida escolar quanto para a vida cotidiana. Mas, para que isso de fato aconteça de forma efetiva e abrangente, é preciso implantar políticas de estado adequadas, que devem ser previstas nos documentos oficiais de educação que regulamentam e direcionam como esta aprendizagem deve acontecer.

Com relação ao ensino do PC na educação básica, esta seção apresenta trabalhos relacionados publicados a partir de 2018, que foi o ano da homologação da BNCC. Barbosa e Maltempi (2020) abordam a necessidade de atualizar a formação inicial de professores em vista de atender as novas diretrizes definidas pela BNCC. Neste trabalho eles relatam a experiência de ensino vivenciada na disciplina de Informática na Educação Matemática ofertada no sétimo semestre do curso de Licenciatura em Matemática de uma universidade pública do estado de São Paulo. Na BNCC, o PC aparece como uma habilidade a ser desenvolvida dentro da disciplina de Matemática. Contudo, em 2019, a maioria dos alunos nunca sequer tinha ouvido o termo. A conclusão dos autores foi que o desconhecimento do assunto é potencialmente maior considerando-se os professores que já se formaram há anos e estão exercendo a profissão. Pasqual Júnior e Oliveira (2019) também mencionam o fato de o PC ser pouco conhecido por professores e acadêmicos e que essa falta de clareza, até mesmo para egressos do curso de Licenciatura em Computação, é uma dificuldade para a atuação docente do ensino deste conteúdo. Eles também mencionam o fato de algumas escolas não considerarem o ensino do PC em seu currículo escolar, mesmo estando proposto na versão final da BNCC. Nascimento et al. (2018) realizaram um mapeamento sistemático, buscando artigos que abordam a oferta do Pensamento Computacional de maneira interdisciplinar na educação básica. O objetivo dos artigos encontrados era mostrar o quanto o ensino do PC pode favorecer no bom desempenho dos alunos nas disciplinas da educação básica e nas provas que avaliam a qualidade de ensino do sistema educacional, como a Prova Brasil. Entretanto, não foi abordado o contexto específico do estado do Paraná.

Desta forma, o diferencial deste trabalho está em apresentar como o PC está sendo apresentado nos documentos oficiais da educação e nas escolas públicas da educação básica do Paraná. Particularmente, um mapeamento da oferta de formação inicial e continuada dos docentes que ministram o PC, a presença do PC nas escolas públicas no estado e esforços regionais complementares.

\section{Legislação Brasileira e Paranaense para o Ensino do Pensamento Computacional na Educação Básica}

A Lei de Diretrizes e Bases da Educação Nacional (LDB) regulamenta e determina o compromisso que a escola precisa ter com a formação ampla do indivíduo. Não basta somente ensinar os conteúdos elementares, é fundamental formar o cidadão 
capaz de compreender a sociedade na qual vive, nos aspectos políticos, sociais, artísticos, morais e tecnológicos (BRASIL, 1996). Pode-se inferir que o PC se manifesta parcialmente na $\mathrm{LDB}$, por meio da formação tecnológica que o aluno do ensino fundamental precisa receber, conforme é apresentado no inciso II do art. 32: "a compreensão do ambiente natural e social, do sistema político, da tecnologia, das artes e dos valores em que se fundamenta a sociedade". Apesar do foco do PC estar em utilizar conceitos da Computação para resolver problemas, é importante que o aluno aprenda a utilizar as tecnologias que o cercam.

Após a criação da LDB e seguindo-a como referência, foram elaborados os Parâmetros Curriculares Nacionais (PCNs). Nestes documentos, também ficou expressa a necessidade de utilizar ferramentas tecnológicas como um instrumento promotor de uma efetiva aprendizagem. Assim como na LDB, os PCNs não apresentam o termo Pensamento Computacional. Entretanto, é possível encontrar o termo Pensamento Lógico, com o propósito de "questionar a realidade, formulando-se problemas e tratando de resolvê-los, utilizando para isso o pensamento lógico, a criatividade, a intuição, a capacidade de análise crítica, selecionando procedimentos e verificando sua adequação" (BRASIL, 1998). O pensamento lógico, a criatividade, a capacidade de análise e a formulação e resolução de problemas são habilidades muito exploradas no PC. Sendo assim, pode-se considerar que os PCNs do ano de 1998 foram os primeiros a apresentar expectativas de ensino próximas das que são apresentadas no PC.

No ano de 2018, foi homologada a versão final da Base Nacional Comum Curricular (BNCC) (BRASIL, 2018). Trata-se de um documento que normatiza e define o conjunto orgânico e progressivo de aprendizagens que são essenciais para todos os alunos da educação básica. Segundo o site do Ministério da Educação, a partir da homologação, os estados e municípios precisariam adequar seus currículos de acordo com os conteúdos e aprendizagens estipulados na BNCC para que a partir do início do ano letivo de 2020 fossem aplicados aos alunos. O PC aparece na BNCC como uma habilidade a ser desenvolvida desde o ensino fundamental dentro da disciplina de Matemática e aprimorada no ensino médio. Em nível nacional, de todos os documentos que regulamentam o ensino da educação básica, a BNCC é o primeiro a apresentar, de fato, o PC em sua totalidade. Apesar de estar vinculado à disciplina de Matemática como uma habilidade a ser desenvolvida, pode-se considerar um passo promissor para a inclusão do tema na educação básica.

Um fato promissor referente ao ensino do PC na educação básica foi a criação das Normas sobre Computação na Educação Básica - Complemento à BNCC (BRASIL, 2021), na qual o Conselho Nacional de Educação (CNE), por meio da Câmara de Educação Básica (CEB), disponibilizou, de 29 de abril de 2021 até 14 de maio de 2021, o documento para consulta pública permitindo sugestões e contribuições para a escrita da sua versão final. As Normas sobre Computação na Educação Básica - Complemento à $\mathrm{BNCC}$ apresentam o $\mathrm{PC}$ como uma unidade temática (eixo), determinando claramente quais são os objetos de conhecimento (objetivos gerais) e habilidades (objetivos específicos) a serem desenvolvidas em cada série do ensino fundamental (anos iniciais e anos finais) e ensino médio. Com essas normas, finalmente o PC deverá receber o protagonismo que merece, ocupando um espaço próprio dentro do currículo ao invés de ser apenas uma habilidade associada a Matemática. 
Já no estado do Paraná, de acordo com a Secretaria da Educação e do Esporte do Paraná ${ }^{1}$, o estado foi o primeiro do Brasil a ter o seu currículo da educação infantil e ensino fundamental unificado à BNCC. Isto aconteceu por meio da criação do Referencial Curricular do Paraná (PARANÁ, 2018). O PC não aparece explicitamente no referencial, mas, é possível reconhecer parcialmente o que se espera no ensino do PC na disciplina de Matemática: "a partir de problematização proposta, o estudante deve, no seu processo de resolução, compreender o conhecimento matemático envolvido e não apenas aprender a aplicar um algoritmo ou uma regra e, assim, permitir a transferência e a intervenção na realidade" (PARANÁ, 2018). Como o Referencial Curricular do Paraná foi realizado por etapas, o ensino médio não foi contemplado na versão consultada nesta pesquisa, pois estava em processo de finalização para atender as mudanças estipuladas na Lei ${ }^{\circ} 13.415 / 2017$, que deve entrar em vigor nas escolas até o início do ano letivo de 2022.

Em 2019, com base no Referencial Curricular do Paraná, o estado do Paraná apresentou o Currículo da Rede Estadual Paranaense (CREP). O CREP serve para orientar o professor do ensino fundamental em relação aos conteúdos, objetos de conhecimento e objetivos de aprendizagem que devem ser trabalhados em cada trimestre do ano letivo (PARANÁ, 2019). O PC não aparece de forma explícita no CREP, embora possa-se inferir que está incluso parcialmente nas competências da disciplina de Matemática para o ensino fundamental anos finais.

Assim, entende-se que o ensino do PC ainda não é uma prioridade na educação básica do Paraná, já que ele não aparece de forma explícita nos documentos oficiais que normatizam a educação básica no estado. A falta de clareza do ensino de PC no Referencial Curricular do Paraná e no Currículo da Rede Estadual Paranaense torna-o não obrigatório, ficando a cargo do professor decidir se vai ensiná-lo aos seus alunos ou não. Vale ressaltar-se que o Referencial Curricular do Paraná para o ensino médio ainda não foi finalizado, devido a reforma do ensino médio. Assim, existe a esperança de que o ensino do PC tenha notoriedade e ocupe um espaço significativo dentre os conteúdos a serem ministrados na última etapa da educação básica.

\section{Status do Ensino do Pensamento Computacional no Paraná}

\subsection{Formação Inicial e Continuada de Professores}

A falta do PC como um conteúdo explícito nos documentos que regulam o ensino básico do Paraná leva ao questionamento de quais seriam os possíveis motivos para não se estabelecer o ensino do PC como um componente curricular obrigatório. Um dos principais motivos é a quantidade insuficiente de professores habilitados para ministrar este conhecimento. A realidade encontrada nas escolas são estagiários ou técnicos atuando nos laboratórios e professores de Matemática que já atuam na escola ministrando o conteúdo sobre o PC.

Os cursos de Licenciatura em Computação (LC) têm a possibilidade de suprir essa deficiência. No Paraná, são seis instituições que ofertam o curso de Licenciatura em Computação e que estão devidamente cadastradas no Cadastro Nacional de Cursos e

\footnotetext{
${ }^{1}$ http://www.educacao.pr.gov.br/Noticia/Parana-e-o-primeiro-estado-ter-curriculo-unificadoBNCC. Acesso: 02/05/2021.
} 
Instituições de Educação Superior (pelo site e-MEC), no momento da escrita deste trabalho. Destes, três têm ofertas gratuitas e três não gratuitas, dois são presenciais e quatro à distância. Entretanto, os formandos nessa área não encontram muito espaço para atuarem nas escolas, já que ainda não existe a obrigatoriedade da contratação desses profissionais para lecionarem disciplinas obrigatórias do currículo. Os profissionais com formação em LC poderiam atuar nos laboratórios de informática das escolas ou ministrando o conteúdo referente ao PC. Além disso, constatou-se que, dos 399 municípios pertencentes ao estado do Paraná, apenas 29 deles oferecem o curso de Licenciatura em Computação, o que representa 7,26\% dos municípios. Esta pequena porcentagem demostra que o curso está em fase de expansão e vai precisar de anos para conseguir atingir uma representatividade expressiva no Paraná.

Uma alternativa é qualificar os professores que já atuam nas escolas na disciplina de Matemática, por meio de formações continuadas. Nesse sentido, a Secretaria de Estado da Educação e do Esporte do Paraná oferece gratuitamente, desde 2019, pelo site Scratch Day 2019², uma série de vídeos explicativos e materiais pedagógicos destinados a professores sobre PC, Algoritmos, Lógica, Scratch e sugestões de jogos para trabalhar em sala de aula. O Ministério da Educação (MEC) também está oferecendo, desde maio de 2020, três cursos gratuitos de capacitação online, por meio do site $\mathrm{AVAMEC}^{3}$, sobre o PC: Introdução ao PC; Aplicações do PC para os anos iniciais do Ensino Fundamental e Aplicações do PC para os anos finais do Ensino Fundamental. É possível que o estado do Paraná também oferte algum outro tipo de formação a respeito do PC exclusivamente para os professores da rede estadual, no formato de aperfeiçoamento e capacitação obrigatória, seguindo o estipulado no plano de carreira do magistério para a progressão salarial. Porém, não foi possível averiguar isso nesta pesquisa.

\subsection{A Presença do PC nas Escolas Públicas do Paraná}

Esta seção apresenta um panorama geral do ensino do PC nas escolas públicas do Paraná. Para reduzir o escopo da análise, buscou-se investigar, por meio dos websites e páginas do Facebook de cada escola que apareceram mais bem classificadas no ranking do Índice de Desenvolvimento da Educação Básica (IDEB) ${ }^{4}$. Isto porque o ensino do PC é uma atividade recente e escolas com menor IDEB possivelmente ainda estão concentrando-se em reforçar outros conteúdos, colocando o PC em segundo plano.

De acordo com o site da Agência Estadual de Notícias (AEN) do Paraná ${ }^{5}$ o estado registrou o maior crescimento do país na nota do IDEB referente ao ano de 2019. A Agência Estadual de Notícias disponibilizou em seu site dois rankings, um com colégios do ensino fundamental anos finais e outro com colégios do ensino médio, listando, respectivamente, os colégios de cada etapa que obtiveram as melhores notas no IDEB do ano de 2019 no estado do Paraná. Contudo, dos vinte e quatro colégios consultados (que apareceram entre os melhores do ensino fundamental anos finais e do ensino médio), apenas o Colégio Estadual do Campo Presidente Kennedy, localizado no

\footnotetext{
2 https://sites.google.com/view/scratch-day-2019/materiais-de-apoio. Acesso: 15/10/2020.

3 https://avamec.mec.gov.br/. Acesso: 15/10/2020.

4 http://ideb.inep.gov.br/resultado/. Acesso em 22/11/2020.

5 http://www.aen.pr.gov.br/modules/noticias/article.php?storyid=108806\&tit=Conheca-oscolegios-estaduais-com-melhores-desempenhos-no-IDEB. Acesso em 22/11/2020.
} 
município de Serranópolis do Iguaçu, apresentou indícios sobre o ensino do PC por meio do compartilhamento de uma postagem do Núcleo Regional de Educação de Foz do Iguaçu sobre um curso on-line de programação gratuito.

A pesquisa, então, estendeu-se a programas estaduais referentes a escolas públicas. Ao consultar o site da Secretaria da Educação e do Esporte $^{6}$ do Paraná, verificou-se que o governo do estado destaca as aulas de programação como uma das inovações presentes nos novos colégios cívico-militares. Em 2020, 199 colégios da rede estadual aprovaram a adoção do modelo cívico-militar, a partir do $6^{\circ}$ ano. Além dos colégios cívico-militares, de acordo com uma matéria publicada na $\mathrm{AEN}^{7}$, o programa Paraná Integral, lançado em 2020 e que consiste na oferta de ensino em período integral, já conta com 17 colégios que ofertam o ensino fundamental anos finais e o ensino médio e a previsão é de que o número de colégios aumente para 34 ainda em 2021. Além de contarem com uma carga horária maior de estudos na escola, os alunos recebem aulas de disciplinas diferenciadas das demais escolas que não aderiram ainda a este formato de ensino integral, incluindo disciplinas de programação. Assim, pode-se inferir que o ensino do PC já acontece nessas escolas, embora, não apareça de forma explícita nos websites das escolas.

Além da oferta de disciplinas de programação em colégios cívico-militares e de tempo integral, a Secretaria da Educação e do Esporte do Paraná, por meio do Programa EDUTECH $^{8}$, oferta de maneira on-line (por meio da plataforma educacional de programação e Ambiente Virtual de Aprendizagem) cursos de tecnologia e inovação aos estudantes do ensino fundamental - anos finais, ensino médio, educação profissional e educação de jovens e adultos da rede pública estadual de ensino e professores pertencentes ao quadro de funcionários. Foram disponibilizadas 66.400 vagas para estudantes e 2.000 vagas aos professores da rede pública estadual. Com duração do ano letivo vigente e apresentando, em média, 150 horas de carga horária, envolvendo games, animações, ciência de dados e programação.

Além do EDUTECH, no site Dia a Dia Educação ${ }^{9}$, da Secretaria da Educação e do Esporte do estado do Paraná, é possível encontrar a definição do que é o Pensamento Computacional e sugestões de atividades e jogos para trabalhar com o PC nas escolas de forma plugada e desplugada. Com essas sugestões de atividades oferecidas pela Secretaria de Educação do Paraná, percebe-se que, embora ainda com atenção insuficiente ao assunto, o estado se preocupa em oferecer recursos pedagógicos aos professores para que apresentem o PC dentro da realidade de cada turma e escola.

Por fim, os colégios também podem contar com o auxílio de universidades ou grupos de pesquisa. Os dois esforços de maior destaque neste sentido no Paraná são o programa Manna Team e o projeto Code Wars. O programa Manna Team ${ }^{10}$, sediado na Universidade Estadual de Maringá, é, atualmente, a maior rede de ensino e pesquisa no

\footnotetext{
${ }^{6}$ http://www.educacao.pr.gov.br/Noticia/Parana-tera-215-colegios-civico-militares-maiorprograma-do-Brasil\#. Acesso em 22/11/2020.

${ }^{7}$ http://www.aen.pr.gov.br/modules/noticias/article.php?storyid=109896\&tit=Escolas-doprograma-Parana-Integral-vao-dobrar-em-2021. Acesso em 03/12/2020.

${ }^{8}$ http://www.educacao.pr.gov.br/programacao. Acesso: 16/11/2021.

9 http://www.educadores.diaadia.pr.gov.br/modules/conteudo/conteudo.php?conteudo=1625. Acesso: 15/102020.

${ }^{10}$ https://manna.team/
} 
campo de Robótica, Internet das coisas e PC do estado. De acordo com o site oficial do programa, desenvolveu em 2019 e 2020 atividades em escolas públicas de 14 municípios paranaenses, atendendo uma estimativa de cerca de 12 mil pessoas. Já o projeto Code Wars ${ }^{11}$ ensina programação em escolas públicas do Paraná desde 2015, com aulas no contraturno e utilizando os laboratórios de informática das instituições.

\subsection{Esforços Regionais Associados ao Ensino de Pensamento Computacional}

Além dos programas mencionados, que atendem de modo geral todo o Paraná, também foi possível encontrar atividades relacionadas ao ensino do $\mathrm{PC}$ em escolas públicas de determinadas regiões. Para isso, fez-se uma análise dos esforços na capital, Curitiba, e em Londrina, a segunda maior cidade do estado, como representante do interior do estado. Além de serem as maiores cidades do estado, também foram as que mais tiveram colégios bem classificados de acordo com a nota do IDEB de 2019.

Em Curitiba, está localizado o Laboratório Pedagógico de Inovação (LAPI) ${ }^{12}$. O LAPI é um ambiente integrado com uma gama de microambientes interdependentes de pesquisa, planejamento, experimentação, modelagem, prototipagem, apresentação de projetos, concepção de ideias e construção de protótipos. Mesmo não aparecendo de forma explícita a oferta do PC no LAPI, pode-se concluir que ele é ensinado por meio da construção de protótipos, do uso dos kits de robótica e de programação, afinal, ao construir um protótipo ou desenvolver um programa, o aluno precisa realizar suas atividades de maneira particionada e solucionar problemas a cada etapa do projeto. Curitiba também desenvolve, desde 2017, o programa Faróis do Saber e Inovação ${ }^{13}$, um projeto que acontece atualmente em 9 praças e 32 escolas da capital. Funcionam em espaços onde antes eram apenas bibliotecas escolares e hoje oferecem computadores com acesso gratuito à internet e atividades extracurriculares por meio das oficinas de mídias digitais; programação; construção de protótipos; modelagem 3D; criação de jogos digitais e de tabuleiro, dentre outras atividades. O objetivo do programa é atender estudantes da rede municipal de ensino de Curitiba no contraturno das aulas regulares e a comunidade que tiver interesse.

Em contraponto, de acordo com as informações encontradas nos veículos de comunicação, Londrina não possui um laboratório de tecnologia destinado aos alunos da rede pública e nem tem em suas escolas Faróis do Saber e Inovação, ou projetos semelhantes, como é ofertado na capital. Ao consultar o site da Secretaria de Educação de Londrina ${ }^{14}$, verificou-se que somente as escolas públicas municipais de educação integral possuem, além das disciplinas do núcleo comum (que são ofertadas em todas as instituições), oficinas pedagógicas, dentre as quais estão a de Matemática e de Formação Socioambiental e Tecnologia. Cria-se uma expectativa de que o PC possa ser ensinado nestas duas oficinas. Além disso, verificou-se apenas dois projetos locais. Uma

\footnotetext{
${ }^{11}$ http://www.matematica.seed.pr.gov.br/modules/noticias/article.php?storyid=945. Acesso:

$24 / 11 / 2020$.

12 https://educacao.curitiba.pr.gov.br/conteudo/laboratorio-pedagogico-de-inovacao-lapi/9860. Acesso: 24/11/2020.

13 https://educacao.curitiba.pr.gov.br/conteudo/farois-do-saber-em-escolas/7363 e https://www.bandab.com.br/cidades/muito-mais-que-bibliotecas-novos-farois-do-saber-setransformam-em-estudios-de-criacao/. Acesso: 10/05/2021.

14 https://portal.londrina.pr.gov.br/todos-artigos/secretarias/sec-educacao/ensinofundamental/ampliacao-de-jornada-escolar. Acesso: 05/12/2020.
} 
escola do município foi atendida pelo programa $\mathrm{WASH}^{15}$, em 2012. O programa WASH tem como objetivo principal a promoção da iniciação científica e a popularização da ciência para estudantes por meio de oficinas que permitem aos estudantes vivenciar conteúdos da Ciência, Tecnologia, Engenharia, Artes e Matemática, por meio do método científico e apoiado na aprendizagem de técnicas de programação de computadores, dentro do contexto das escolas da rede pública. Por fim, a Universidade Estadual de Londrina, por meio do programa Robótica Educacional para Meninas e Jovens $^{16}$, dirigido por estudantes de engenharias da instituição, busca motivar alunos do ensino médio a escolherem carreiras de engenharia. O objetivo geral do programa é transferir o conhecimento básico de engenharia de forma lúdica, a partir da robótica educacional e da construção de protótipos criados a partir de sucatas. Apesar do objetivo não ser o ensino do PC, possivelmente este conteúdo é ensinado ao construir os protótipos e utilizar a robótica educacional.

Comparando a capital com o interior do estado, no que se refere a oferta do PC de forma direta ou indireta, Curitiba apresentou informações concretas sobre o LAPI e os Faróis do Saber e Inovação, mostrando o quanto esses dois projetos estão bem consolidados. Por outro lado, com relação a Londrina, não há informação sobre a quantidade de alunos ou escolas contempladas pelo projeto Robótica Educacional para Meninas e Jovens e o projeto WASH foi disponibilizado em apenas uma escola do município, mostrando que o interior está bastante aquém da capital.

\section{Conclusão}

O ensino do PC na educação básica está amparado por meio da BNCC como uma habilidade a ser desenvolvida obrigatoriamente desde o início do ano letivo de 2021, no ensino fundamental e médio nas aulas de Matemática. Já o documento com as Normas sobre Computação na Educação Básica - Complemento à BNCC, em fase de finalização, apresentam o PC em sua totalidade, evidenciando-o como um conteúdo oriundo da Computação e não uma habilidade incorporada a disciplina de Matemática, como foi apresentado na BNCC. Entretanto, como o próprio título diz, as Normas são um complemento. Portanto, não se sabe ao certo se serão agregadas à $\mathrm{BNCC}$ e se tornarão obrigatórias ou se ficará facultativa a sua inclusão nos currículos das escolas.

Os resultados desta pesquisa mostram que o termo Pensamento Computacional não aparece de forma explícita nos documentos que regulamentam a educação básica do Paraná. O PC também não aparece explicitamente nos programas que a Secretaria de Estado da Educação e do Esporte do Paraná oferece de forma extracurricular. A oferta de disciplinas de programação e tecnologia computacional nos colégios estaduais em tempo integral permite inferir que o PC é abordado nestas escolas, ainda que indiretamente, mas o número de escolas atendidas é muito reduzido. $\mathrm{O}$ cumprimento da BNCC referente ao ensino do PC no estado do Paraná permanece como uma incógnita. Sua efetividade, aparentemente, acontecerá a depender do esforço do próprio professor em sala de aula, já que legalmente o PC não aparece de forma explícita nos documentos oficiais da educação do Paraná e nem de forma extracurricular pelos programas oferecidos pelo estado. O que existem são expectativas de que seu ensino aconteça,

\footnotetext{
${ }^{15}$ https://wash.net.br/2020/09/08/jogando-para-pensar-pesquisa-leva-pensamentocomputacional-as-criancas-do-ensino-fundamental/\#. Acesso: 05/12/2020.

${ }^{16} \mathrm{http}: / /$ www.uel.br/projetos/robotica/?page id=10. Acesso: 05/12/2020.
} 
mesmo que superficialmente, no decorrer de determinadas disciplinas ou por meio de programas independentes, que não são comandados pela secretaria de ensino do estado.

Agradecimentos: Este trabalho teve apoio da CAPES e do CNPq.

\section{Referências}

Arruda, E. P. (2017) Documento técnico contendo estudo sobre o processo de implementação das tecnologias digitais nos currículos das escolas de educação básica dos Países membros da OCDE, Organização das Nações Unidas para a Educação, a Ciência e a Cultura, Conselho Nacional de Educação, Brasília.

Barbosa, L. L. S.; Maltempi, M. V. (2020) Matemática, Pensamento Computacional e BNCC: desafios e potencialidades dos projetos de ensino e das tecnologias na formação inicial de professores. Revista Brasileira de Ensino de Ciências e Matemática, v. 3, n. 3, 748-776.

Brasil. (1996) Lei de diretrizes e Bases da Educação Nacional. Lei no 9.394/1996, Brasília, DF.

Brasil. (1998). Ministério da Educação e Cultura. Secretaria de Ensino Fundamental. Parâmetros Curriculares Nacionais. Brasília, DF.

Brasil. (2018) Ministério da Educação. Base Nacional Comum Curricular. Brasília, DF.

Brasil. (2021) Ministério da Educação. Conselho Nacional de Educação. Normas sobre Computação na Educação Básica - Complemento à BNCC. Distrito Federal.

Moran, José Manuel (2007). A educação que desejamos: Novos desafios e como chegar lá. Campinas, SP: Papirus.

Nascimento, C.; Santos, D. A.; Tanzi, A. (2018) Pensamento computacional e interdisciplinaridade na educação básica: um mapeamento sistemático. In: Anais dos Workshops do Congresso Brasileiro de Informática na Educação. p. 709-718.

Paraná. (2018) Secretaria de Estado da Educação. Referencial curricular do Paraná: princípios, direitos e orientações. Curitiba, PR.

Paraná. (2019) Secretaria de Estado da Educação. Currículo da Rede Estadual Paranaense - CREP. Curitiba, PR.

Pasqual Júnior, P. A.; Oliveira, S. (2019) Pensamento Computacional: Uma Proposta de Oficina Para a Formação de Professores. Revista Novas Tecnologias na Educação, v. 17, p. 62-71.

SBC. (2019) Diretrizes para o ensino de computação na educação básica. Sociedade Brasileira de Computação. https://www.sbc.org.br/educacao/diretrizes-para-ensinode-computacao-na-educacao-basica

Wing, J. (2006) Pensamento computacional - Um conjunto de atitudes e habilidades que todos, não só cientistas da computação, ficaram ansiosos para aprender e usar. Revista Brasileira de Ensino de Ciência e Tecnologia, v. 9, n. 2.

Wing, J. (2014) Computational Thinking Benefits Society. Social Issues in Computing, New York: Academic Press. 